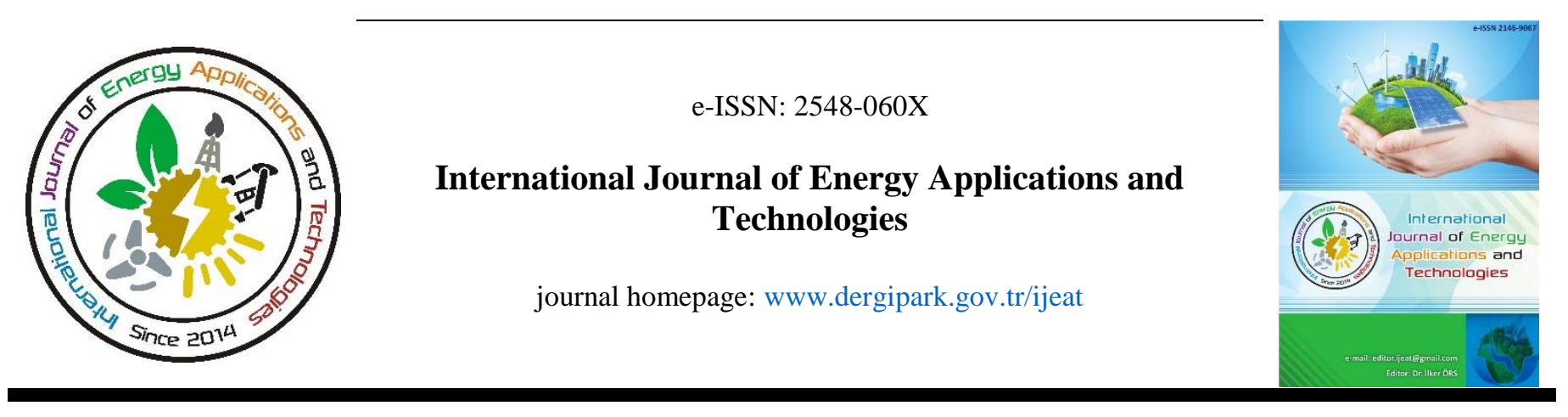

Original Research Article

\title{
A study on the effect of blade inlet angle, attack angle and the diameter ratio on the efficiency of a Banki Mitchell turbine
}

\author{
Niyigena Hirwa Jean Paul \\ Department of Energy Systems Technology Engineering,University of Kocaeli, Kocaeli,Turkey
}

\author{
ARTICLE INFO \\ * Corresponding author \\ niyihirwa45@gmail.com \\ Received September 21, 2021 \\ Accepted November 10, 2021 \\ Published by Editorial Board \\ Members of IJEAT \\ (C) This article is distributed by \\ Turk Journal Park System under \\ the CC 4.0 terms and conditions. \\ doi: 10.31593/ijeat.998333
}

\begin{abstract}
In the present work, a theoretical investigation is presented on how the efficiency of a Banki Mitchell turbine is affected by its design parameters. With the help of general formulation of the control volume theory to the equation of the moment of momentum in the turbine's runner and considering all assumption regarding the properties of the flow and the design geometry of the runner, a mathematical model has been developed. The water discharge through the turbine is considered incompressible, non-viscous, homogenous and steady flow; The loss due to shock on the inner and outer surface of the runner as well as leakage losses are neglected. As results of the present study, the behaviour of hydraulic efficiency was determined when varying the angle of attack, the blade entry angle as well as the runner diameter ratio, indicating their appropriate values in each different scenario. In this paper critical relationships between design parameters have been disclosed and therefore analysed and simulated using Simulink MATLAB. Furthermore, it was mentioned that for a better performance, the exit angle of the blade should be $90^{\circ}$. At length, an expression was given estimating the turbine's efficiency at various operating conditions and for calculating the energy contribution per stage, respectively.
\end{abstract}

Keywords: Banki Mitchell Turbine, Fluid Mechanics Analysis, Control Volume Theory, Efficiency

\section{Introduction}

In recent years, small-scale hydrogeneration has become a good alternative for exploiting renewable energy resources in a clean way. To make the most of water resources, it is necessary to look for low-cost alternatives compared to conventional hydroelectric facilities. For this purpose, the cross-flow turbine is the one that is best suited, due to its good performance in small-scale power generation, its simple design, low manufacturing and maintenance cost.

The cross-flow turbine, which is also called the BankiMitchell or Ossberger turbine, is classified as an impulse turbine which is mostly used in smaller hydropower plants with power output range $5-100 \mathrm{KW}$ [1]. Cross flow turbine consists of two main elements: an injector and a runner; The injector is that tubular element of rectangular section that conducts the flow towards the runner at a constant angle with respect to the tangent to the flow path and the runner is made up of two parallel discs that are joined by the edges by means of a set of curved blades in the shape of a circular arc. The energy transferred to the turbine runner is carried out in two stages where an average of $68.5 \%$ is contributed in the first stage and $31.5 \%$ in the second [2]. On the other hand, the flow through the runner has been analysed by Banki many years ago considering an impulse turbine with a representative current line through a one-dimensional analysis [3]. The flow inside this type of turbine is very complex because the water partially passes through the runner, making the flow unstable [4]. In the present study, a theoretical analysis of the hydraulic efficiency in the runner of a cross-flow turbine is carried out by varying certain design parameters $\left(\alpha_{1}, \beta_{1}, D_{2} / D_{1}\right)$ linked to it, in addition, the hydraulic efficiency of the turbine is estimated at different regimes of $\mathrm{n}_{11}$, as this will allow to know a priori the 
performance of the runner before being manufactured. For this purpose, we apply the method of the control volume to the equation of the moment of the momentum, considering some hypotheses about the characteristics of the flow as well as the geometry of the runner; also finding some optimal relationships between geometric parameters of the runner that maximize hydraulic efficiency, as well as an expression that allows calculating the energy contribution per stage.

\section{Material and Methods}

\subsection{General considerations}

The cross-flow turbine under study is considered a free-jet action turbine, which means that, it works at a constant pressure equal to atmospheric pressure. The flow that crosses through the runner has been considered non-viscous, homogeneous, incompressible and in steady state; furthermore, it is assumed that water flow completely passes through the runner. The velocity triangles of the fluid particles are also considered to be equal in the circumferential direction. Load losses in the runner blades as well as leakage losses are neglected. It is considered that the runner has as many blades as necessary, so that the space between blades may be signifyingly small as required, such that the circulation of any fluid particle is zero and therefore the flow is irrotational. The runner blades are assumed to be geometrically identical and their surfaces free of roughness. Shock losses on the outer and inner periphery of the runner are neglected. According to this assumption, there are no shock losses at the entrance of each of the stages, and assuming the presence of a quantity of blades as large as required, in Figure 1 the fluid particle that passes through point $\mathrm{X}$ of the streamline must have the relative component of the velocity, coinciding with the direction of the tangent to the blade. Therefore, we can generalize this fact for each point of the portions of current lines that make contact with the runner blades.

Therefore, the equation that will be used to compute the torque that the fluid exerts on the turbine's blades, is found by applying the general equation of the control volume method to the equation of moment of the momentum [5] and we obtain:

$\vec{M}=\int_{C A} \vec{r} x \vec{V} \cdot(\rho \vec{V} \cdot \vec{n}) d A+\frac{\partial}{\partial t} \int_{C V} \vec{r} x \vec{V} \rho d V$

As mentioned above since we assume our flow to be a steady flow, the amount of angular momentum within the control volume remains constant, and thus the time rate of change of angular momentum of the contents of the control volume is zero [5]. resulting in:

$\vec{M}=\int_{C A} \vec{r} x \vec{V} \cdot(\rho \vec{V} \cdot \vec{n}) d A$
As the control area is in turn composed of four surfaces as shown in the points of water flow when it passes through any blade; From what has already been mentioned, we can deduce that the upper end points X1 and X2 through which the flow enters could coincide with the ends of two runner blades at a certain moment, thus in the same way the ends Y1 and $\mathrm{Y} 2$ through which the flow exits could coincide with the lower ends of two other blades. Finally, it is assumed that the flow does not impact the turbine runner shaft and that the turbine runner is in uniform rotary motion.

\subsection{Mathematical modelling}

For the development of the mathematical model, a control volume has been considered as, which is delimited by two concentric cylindrical surfaces that contain the inner and outer surfaces of the runner as well as by two lateral surfaces, superimposed on the inner side of the circular discs of the runner limiting the lateral space contained between the inner and outer periphery of the runner, in this way we will obtain a control volume in the shape of a hollow solid cylinder shown in Figure 2. This control volume is attached to an inertial reference system located on the runner shaft, in addition, the components of speed as well as acceleration will be assumed to be null in the axial direction, so the flow movement is reduced to a movement in the plane transverse to the axis.

And since Figure 2, the velocity field on the lateral surfaces A3 and A4 of the control volume is perpendicular to the vector normal to these surfaces, then the expression is obtained:

$$
\vec{M}=\int_{A 1} \vec{r} x \vec{V} \cdot(\rho \vec{V} \cdot \vec{n}) d A+\int_{A 2} \vec{r} x \vec{V} \cdot(\rho \vec{V} \cdot \vec{n}) d A
$$

With: A1 and A2, the exterior and interior surface area of the control volume respectively.

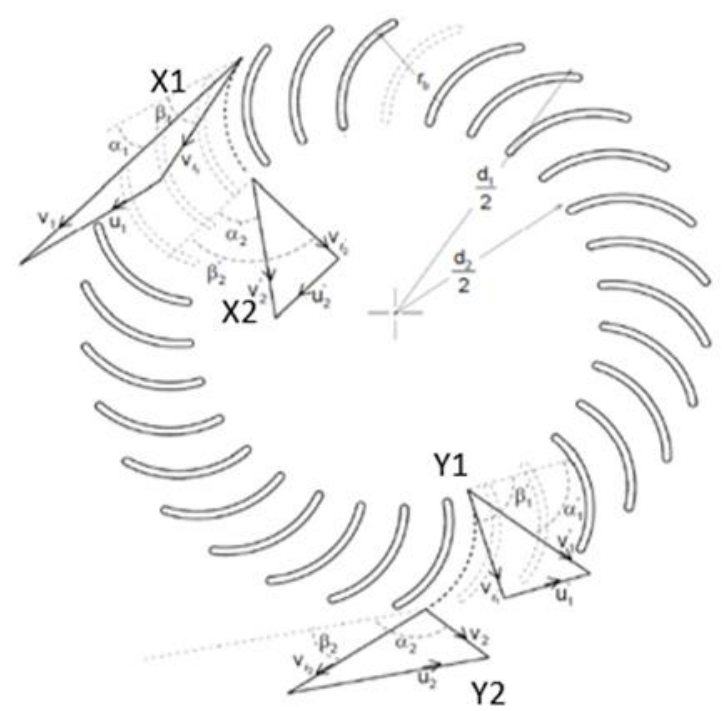

Fig. 1. Kinematic flow configuration in the rotor of the cross-flow turbine 
Finally, taking into account that the flow is homogeneous and incompressible, the expression of the torque in the axial direction is obtained:

$M=\left(\rho B v_{2}^{2} R_{2}^{2} \theta \sin \alpha_{2} \cos \alpha_{2}-\rho B v_{1}^{2} R_{1}^{2} \theta \sin \alpha_{1} \cos \alpha_{1}\right)+$ $\left(\rho B v_{4}^{2} R_{1}^{2} \theta \sin \alpha_{4} \cos \alpha_{4}-\rho B v_{3}^{2} R_{2}^{2} \theta \sin \alpha_{3} \cos \alpha_{3}\right)$

Where it can be seen that the effective torque exerted on the flow can be considered as the sum of the individual torque effected in each of the stages.

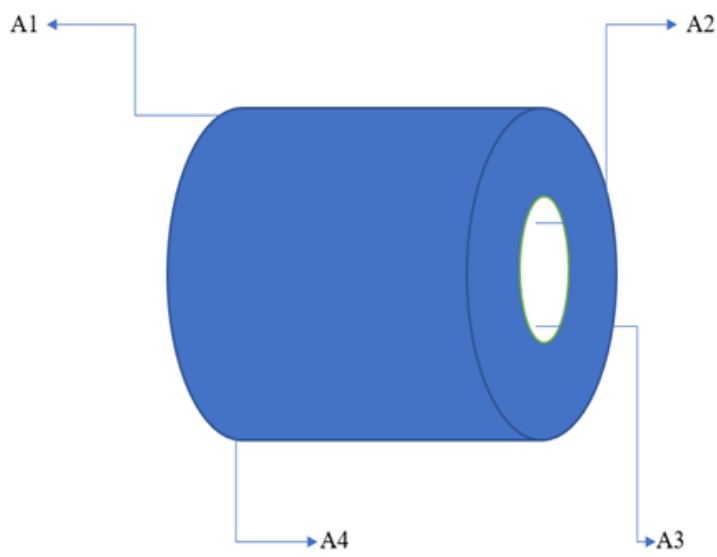

Fig.2. Schematic of the control volume with its conformant control surfaces

In the kinematic configuration of the flow, as shown in Figure 1 , a schematic current line can be seen in the region of doted blades, this in turn is the trajectory of a fluid particle because it is the steady flow. If we join the points of the current line that intersect the inner periphery of the runner with the centre of the runner, these will form an isosceles triangle, since the section of the current line inside the runner can be assumed to be practically rectilinear; also considering that there are no collision losses in the internal and external periphery of the runner, the fluid particle that enters the second stage will have a relative component in the same direction as the tangent of the blade at that moment and neglecting the increase in speed due to the difference in level between the output of the first stage and the input of the second stage, it follows that:

$\alpha_{2}=\alpha_{3}$

$\beta_{2}=\beta_{3}=90^{\circ}$

$v_{2}=v_{3}$

$w_{2}=w_{3}$

And since it is assumed that there is no friction between the flow and the blades, then:

$w_{1}=w_{4}$

Thus, the velocity triangles at the input and output of each stage are as shown in Figure 3, and therefore equation (4) becomes:

$$
M=\left(\rho B v_{4}^{2} R_{1}^{2} \theta \sin \alpha_{4} \cos \alpha_{4}-\rho B v_{1}^{2} R_{1}^{2} \theta \sin \alpha_{1} \cos \alpha_{1}\right)
$$

By the volumetric continuity equation between the flow inlet and outlet, it is concluded that:

$$
v_{4} \sin \alpha_{4} B L_{4}=\mathrm{v}_{1} \sin \alpha_{1} B L_{1}=\mathrm{Q}
$$

What's more:

$$
\mathrm{L}_{1}=\mathrm{L}_{4}=\mathrm{R}_{1} \cdot \theta
$$

Considering the runner with uniform rotary motion, the power transferred by the flow to the runner will be given by the following expression:

$$
\mathrm{Pe}=-\mathrm{M} . \omega
$$

Replacing equations (6), (7) and (8) in (9) the final expression is obtained for the calculation of the power exerted on the runner shaft:

$$
\mathrm{Pe}=\rho \mathrm{Q} u_{1}\left(\mathrm{v}_{1} \cos \alpha_{1}-\mathrm{v}_{4} \cos \alpha_{4}\right)
$$

Applying the modified Bernoulli equation between the inlet of the pressure pipe and the outlet of the injector, and considering the speed at the injector outlet equal to the speed at the runner inlet, the latter can be calculated as:

$$
v_{1}=\varphi \sqrt{2 g H}
$$

Experimental studies indicate that the value of $\varphi$ ranges between 0.91 and 0.971 [6].

The turbine Hydraulic efficiency will result from the ratio between the turbine's shaft power and water flow's power and it will therefore be calculated as:

$$
\eta_{h}=\frac{\mathrm{Pe}}{\rho g Q H}
$$

Replacing equations (10) and (11) in (12), the latter becomes:

$$
\eta_{h}=\frac{\pi \varphi}{15 \sqrt{2 g}} \mathrm{n}_{11}\left(\cos \alpha_{1}-\frac{\pi}{60 \varphi \sqrt{2 g}} n_{11}\right)
$$
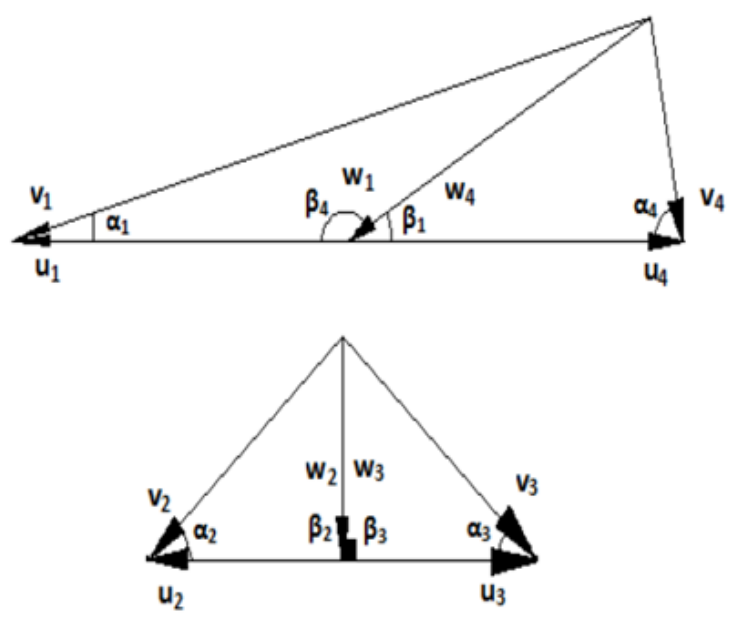

Fig. 3. Velocity triangle in the rotor of the cross-flow turbine

From the velocity triangle in Figure 3, it is obtained that:

$$
\begin{aligned}
& v_{4} \cos \alpha_{4}=2 u_{1}-v_{1} \cos \alpha_{1} \\
& w_{1} \cos \beta_{1}=v_{1} \cos \alpha_{1}-u_{1} \\
& v_{1}^{2}=w_{1}^{2}+u_{1}^{2}+2 w_{1} u_{1} \cos \beta_{1}
\end{aligned}
$$


Replacing equations (10), (11), (14), (15) and (16) in equation (12), we obtain that:

$$
\eta_{h}=4 \varphi^{2} \cdot \frac{\left(\frac{u_{1}}{w_{1}}\right) \cos \beta_{1}}{1+\left(\frac{u_{1}}{w_{1}}\right)^{2}+2\left(\frac{u_{1}}{w_{1}}\right) \cos \beta_{1}}
$$

Taking into account the sine theorem in the velocity triangle of figure 3 , we get the expression of equation (18) which can be written as:

$$
\eta_{h}=4 \varphi^{2} \cdot \frac{\sin \alpha_{1} \cdot \cos \beta_{1} \cdot \sin \left(\beta_{1-} \alpha_{1}\right)}{\sin ^{2} \beta_{1}}
$$

Deriving equation (18) with respect to $u_{1} / w_{1}$ and equating it to zero, we obtain:

$$
\frac{\partial \eta_{h}}{\partial\left(\frac{u_{1}}{w_{1}}\right)}=4 \varphi^{2} \cdot \frac{\cos \beta_{1}\left(1-\left(\frac{u_{1}}{w_{1}}\right)^{2}\right)}{\left(1+\left(\frac{u_{1}}{w_{1}}\right)^{2}+2\left(\frac{u_{1}}{w_{1}}\right) \cos \beta_{1}\right)^{2}}=0
$$

The solution to the equation is obtained when:

$$
\frac{u_{1}}{w_{1}}=1
$$

A direct consequence is obtained from the above equation, where from the velocity triangle in Figure 3 it follows that:

$$
\beta_{1}=2 \alpha_{1}
$$

Only this maximum condition will be adopted since when it is derived with respect to $\beta_{1}$, it is obtained that $\beta_{2}$ must be 0 , which is impossible because the inflow of the flow would be tangential according to (21), thus violating the operating principle of this type of turbines. Therefore, the formula for maximum hydraulic efficiency results:

$$
\eta_{\text {hmax }}=2 \varphi^{2} \cdot \frac{\cos \beta_{1}}{1+\cos \beta_{1}}
$$

Applying the volumetric continuity equation in the first stage, we have:

$$
w_{2}=w_{1} \sin \beta_{1}\left(\frac{D_{1}}{D_{2}}\right)
$$

From the uniform rotary motion, it results:

$$
u_{2}=u_{1}\left(\frac{D_{2}}{D_{1}}\right)
$$

Applying some of the general equations of turbomachines to the first stage, the following relationships are obtained:

$$
\begin{aligned}
& \mathrm{W}=\frac{P_{1}-P_{2}}{\rho}+\frac{v_{1}^{2}-v_{2}^{2}}{2}+\mathrm{g}(\mathrm{z} 1-\mathrm{z} 2)-\left|W_{r}\right| \\
& \mathrm{W}=\frac{w_{2}^{2}-w_{1}^{2}}{2}+\frac{v_{1}^{2}-v_{2}^{2}}{2}+\frac{u_{1}^{2}-u_{2}^{2}}{2}
\end{aligned}
$$

Having assumed the turbine under study as a free jet action turbine, we have: $\mathrm{P}_{1}=\mathrm{P}_{2}=\mathrm{P}_{\mathrm{atm}}$, neglecting the height between the inlet and outlet of each stage as well as the friction losses in the blades $\left(\left|W_{r}\right|=0\right)$; then replacing equations (20), (23) and (24) in (26) and finally equating the relations (25) and (26), the following important relation is obtained:

$$
\frac{D_{1}}{D_{2}}=\sqrt{\sin \beta_{1}}
$$

\section{Results and Discussion}

With the help of Simulink/MATLAB software, the equation (18) and (13) were solved directly by using the model and various outputs were discussed below.

\subsection{Hydraulic Efficiency Curves}

Figure 5 shows the theoretical behaviour of the hydraulic efficiency of the runner of a cross flow turbine with respect to the unit rotational speed for certain values of $\alpha_{1}$, considering a value for $\varphi$ of 0.95 which is very acceptable for this type of turbines [6]. Furthermore, it is observed that the maximum points of hydraulic efficiency are higher the smaller the values of the flow angle $\alpha_{1}$.

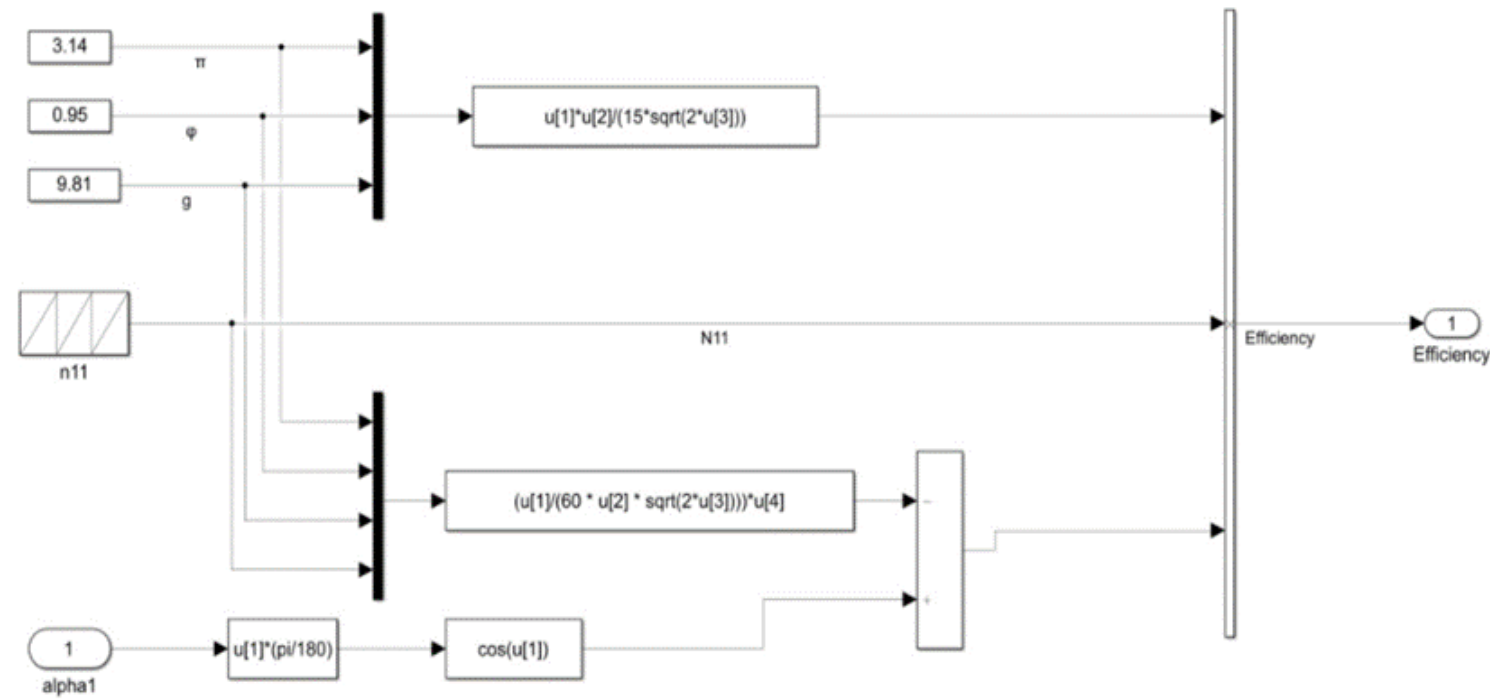

Fig. 4. MATLAB/Simulink for equation (13) 
It can be seen that the hydraulic efficiency values are almost coincident in the different efficiency curves for a unit rotation speed range from 0 to 10 , which is also verified in experimental results [2]. Furthermore, the maximum efficiency points are shifting to the left as the value of $\alpha_{1}$ increases; the growth of the efficiency curve can be clearly observed when the values of $\alpha_{1}$ are decreasing, which is quickly deduced from (18). On the other hand, the maximum points for each particular value of $\alpha_{1}$ have a parabolic behaviour, which is deduced by deriving (13) with respect to $\mathrm{n}_{11}$ and equating to zero, obtaining the following expression:

$$
\eta_{\mathrm{hmax}}=\frac{\pi^{2}}{1800 g} n_{11}^{2}
$$

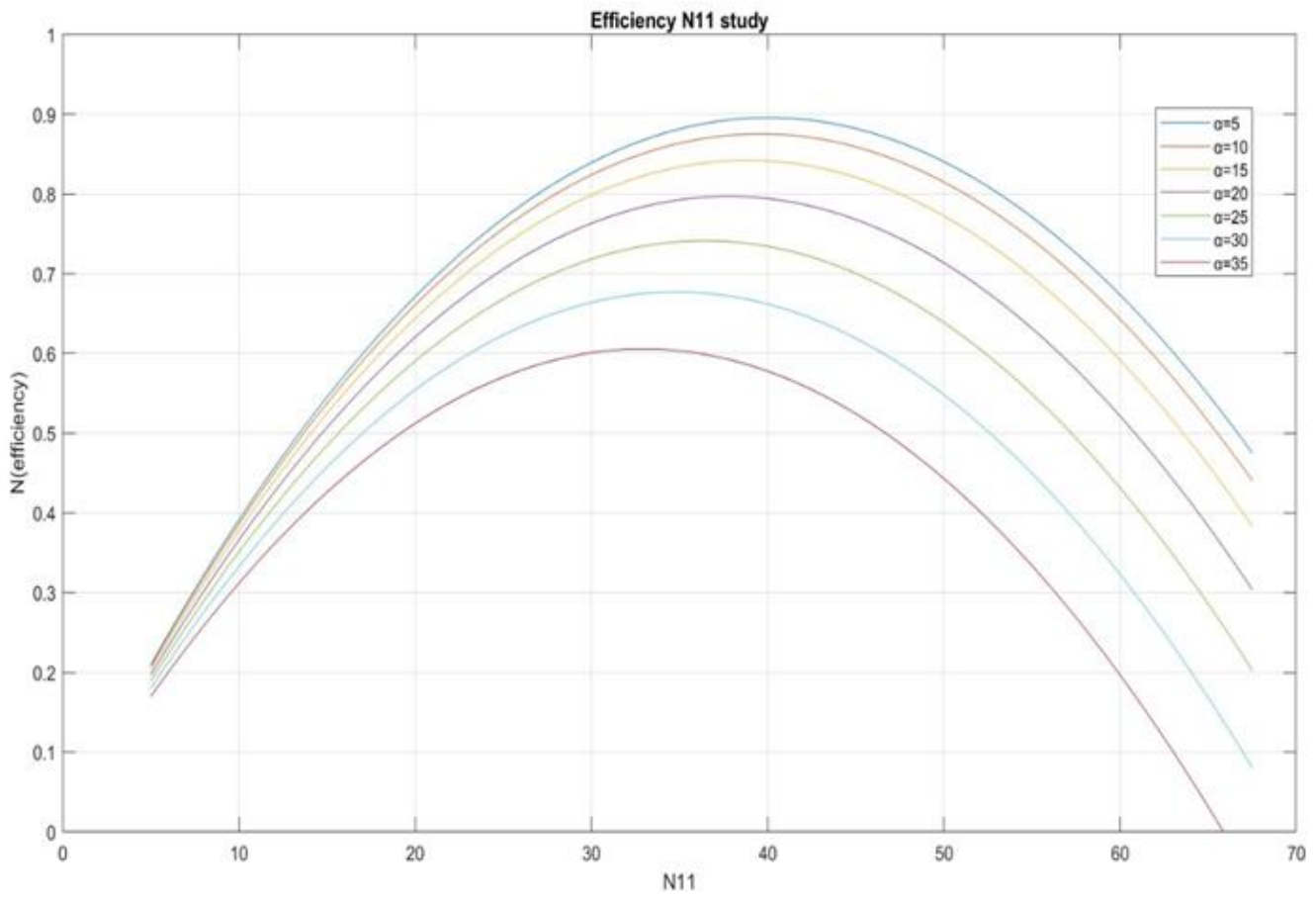

Fig. 5. Hydraulic Efficiency Curves

\subsection{Effect of attack angle $\alpha_{1}$}

Figure 7 shows the behaviour of hydraulic efficiency with respect to the variation of the angle of attack $\alpha_{1}$ at different values of $\beta_{1}$, with a value for $\varphi$ of 0.95 [6]. It can be clearly seen that the maximum values of hydraulic efficiency are obtained when $\alpha_{1}$ is half of $\beta_{1}$ according to what was deduced in (21), in addition the maximum points of efficiency follow a behaviour that is shown with a dashed line decreasing according to what was deduced in (22). It can be seen that for a given value of $\alpha_{1}$ the hydraulic efficiency increases as the value of the angle $\beta_{1}$ decreases; it is also seen that the values of $\alpha_{1}$ corresponding to the maximum efficiencies decrease as the value of the angle $\beta_{1}$ decreases.

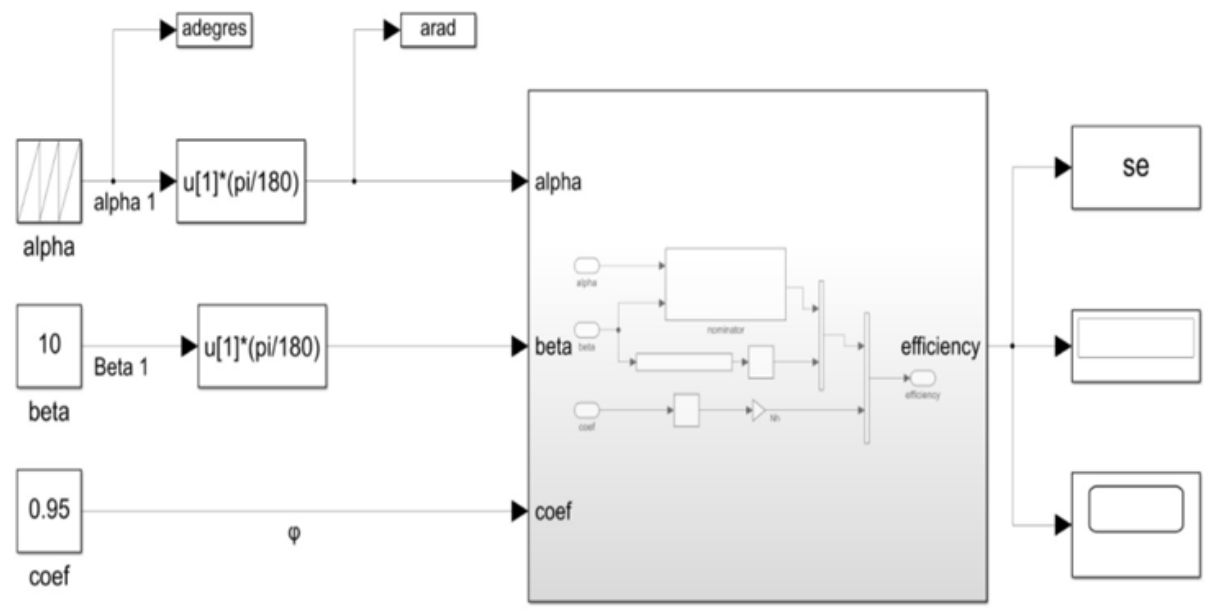

Fig. 6. MATLAB/Simulink for equation (18) 


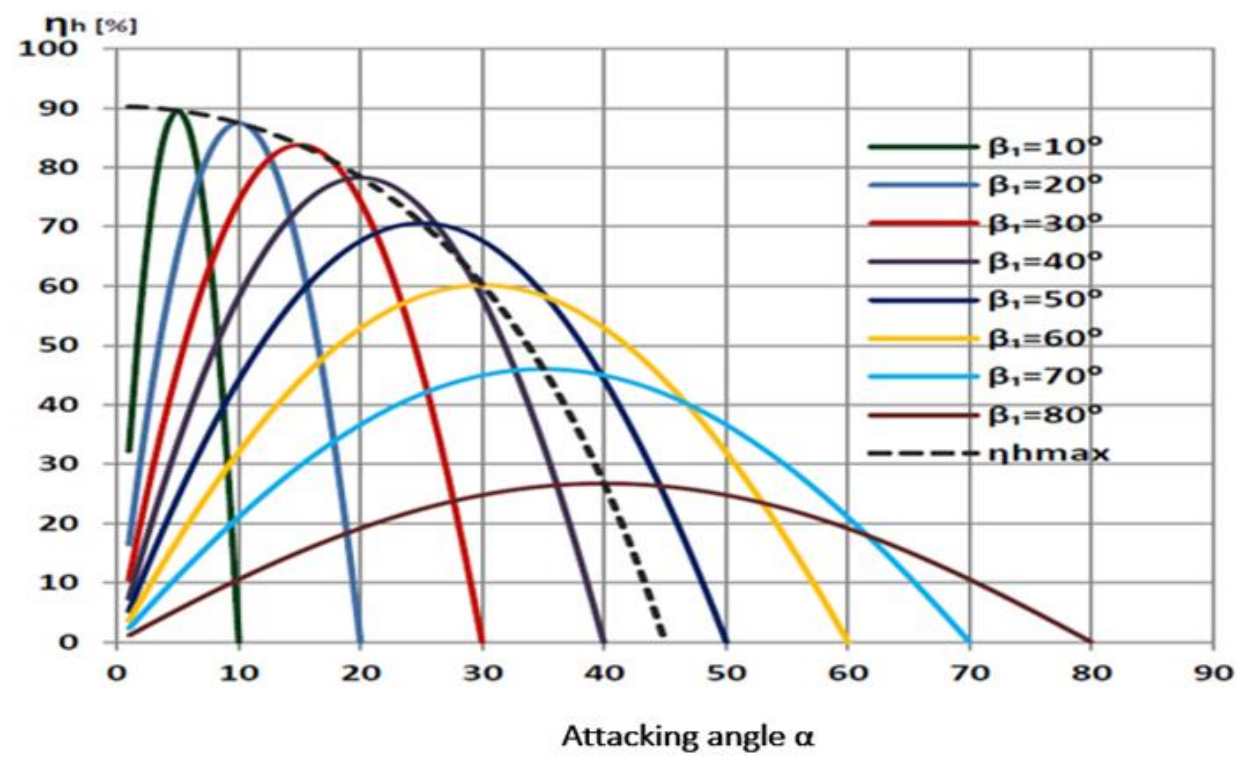

Fig. 7. Hydraulic performance curves as a function of $\alpha_{1}$

It is also appreciated that the maximum points of hydraulic efficiency are higher the smaller the values of the flow angle $\alpha_{1}$ are. These results are also verified in the experimental studies by Desai and Aziz [10] where it is concluded that the greater the angle of attack corresponds to a decrease in efficiency, also Choi and Kim [7] verify the same behaviour in a study when investigating the effect of the configuration. Turbine structural performance and internal flow characteristics through computational modelling.

\subsection{Effect of blade entry angle $\beta_{1}$}

Figure 9 shows the dependence of hydraulic efficiency with respect to the blade entry angle $\beta_{1}$, for different values of $\alpha_{1}$, with a value for $\varphi$ of 0.95 [6]. It can be clearly seen that some maximum efficiency values are obtained when $\beta_{1}$ is very close to twice $\alpha_{1}$, in addition the maximum points of hydraulic efficiency follow a behaviour that is shown with a red dotted line and the maximum points calculated from according to (22) they are joined with a black dashed line. It can be seen that for a given value of $\beta_{1}$ the hydraulic efficiency increases as the value of the angle $\alpha_{1}$ decreases, in addition it can be seen that the values of $\beta_{1}$ corresponding to the maximum efficiencies decrease when the value of the angle $\alpha_{1}$ decreases, and it is also appreciated that the maximum points of hydraulic efficiency are greater the smaller the values of the entry angle of the blade $\beta_{1}$ are. The divergence between the maximum points obtained from (22) and those that emerge from (18) is also clearly appreciated.

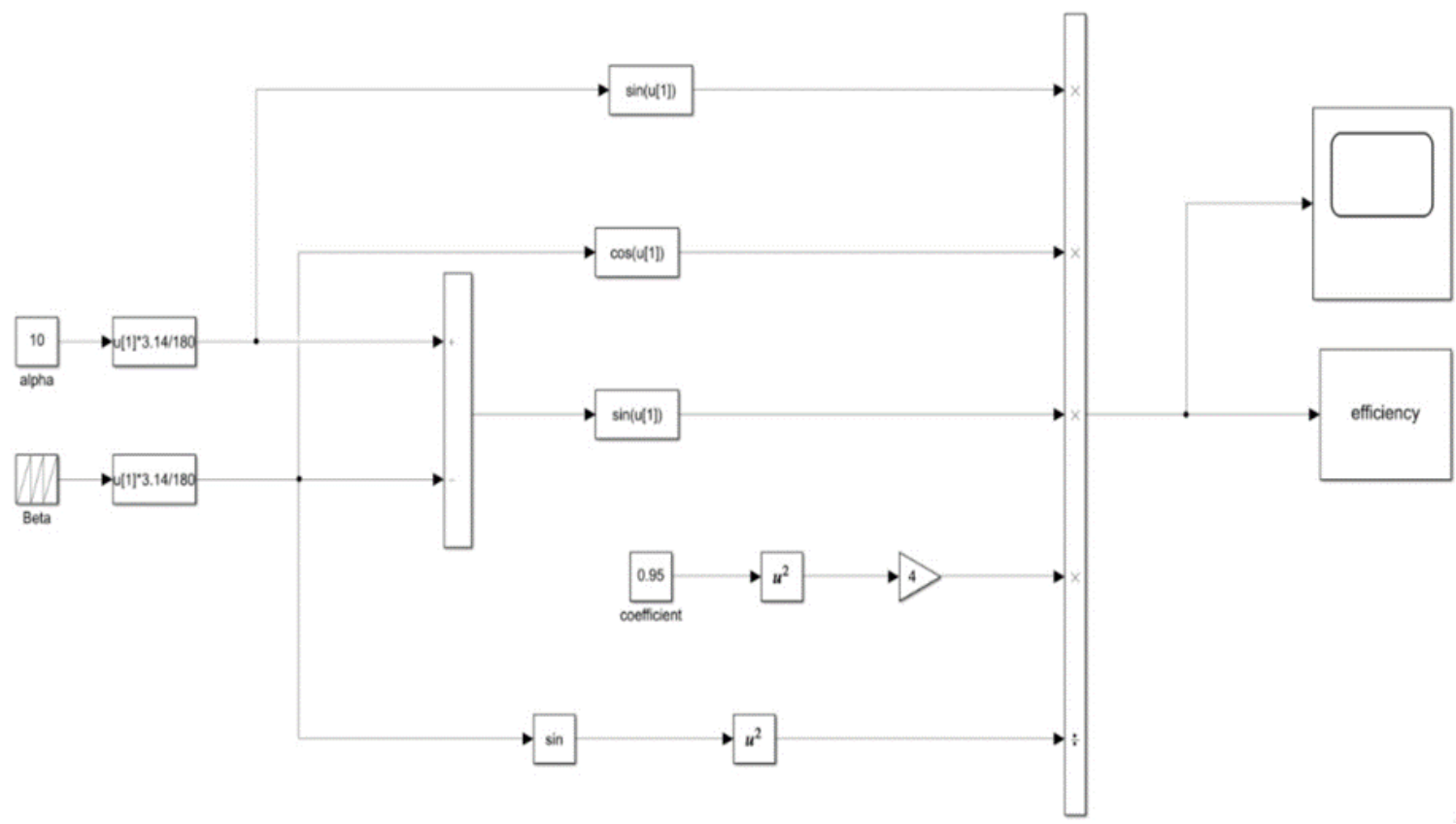

Fig. 8. MATLAB/Simulink for equation (18) 


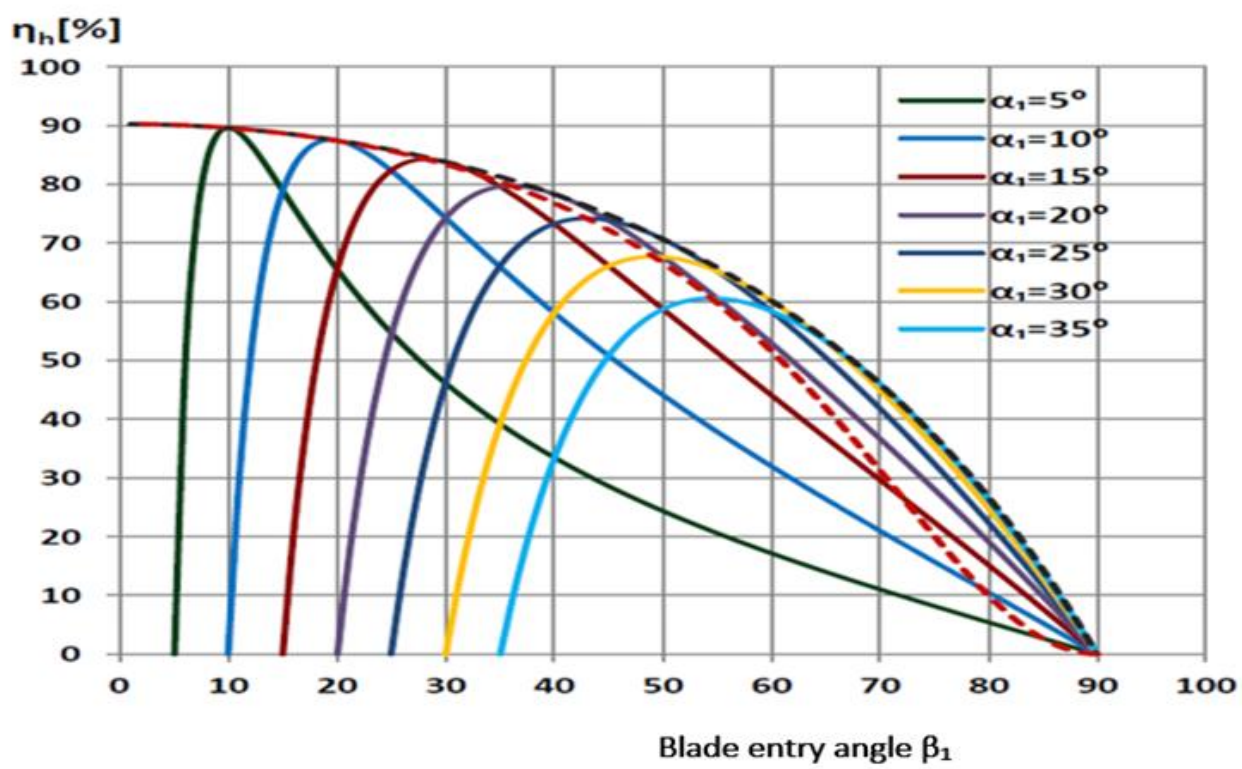

Fig. 9. Hydraulic performance curves as a function of $\beta_{1}$

This is explained because expression (22) was deduced accepting only condition (20), however we see that there is a very good approximation between them when $\beta_{1}$ does not exceed $40^{\circ}$, where both lines practically overlap, which gives us reveals an important result from the theoretical point of view, since we can reach the maximum values of hydraulic efficiency only considering condition (21); then it is clear that once the value of $\alpha_{1}$ has been chosen, then the value $\beta_{1}$ will have to be twice that of $\alpha_{1}$ to reach the maximum efficiency or given the value of $\beta_{1}$, the efficiency is maximized when $\alpha_{1}$ is half of $\beta_{1}$; and the relevance of choosing a not excessively large value for $\alpha_{1}$ is verified again, which according to the graph it would be convenient not to exceed $20^{\circ}$, which is corroborated with values used in practice $^{1}$, which allows us to obtain an acceptable performance in the turbine. Choi and kim [7] conf results the trend of optimal efficiency which decreases as the value of the entry angle of the blade $\beta_{1}$ increases. which is corroborated with values.

\subsection{Energy contribution by stage $\left(\xi_{1}, \xi_{2}\right)$}

Since the net torque effected on the flow can be expressed as the sum of the torque contribution in each of the stages according to (5), the torque calculated in each stage taking into account the expressions (4), (7) and (8) results:

$$
\left.\begin{array}{l}
M_{e-1}=\rho \mathrm{Q}\left(\mathrm{R}_{1} \mathrm{v}_{1} \cos \alpha_{1}-\mathrm{R}_{2} \mathrm{v}_{2} \cos \alpha_{2}\right) \\
M_{e-2}=\rho \mathrm{Q}\left(\mathrm{R}_{2} \mathrm{v}_{3} \cos \alpha_{3}-\mathrm{R}_{1} \mathrm{v}_{4} \cos \alpha_{4}\right)
\end{array}\right\}
$$

After considering the angle between absolute velocity and tangential velocity equivalent for both stage one and stage two, after also considering the absolute velocity equivalent for both stages, the total torque results:

$M_{e}=\rho \mathrm{Q}\left(\mathrm{R}_{1} \mathrm{v}_{1} \cos \alpha_{1}-\mathrm{R}_{1} \mathrm{v}_{4} \cos \alpha_{4}\right)$
The energy contribution coefficients per stage are given by: Figure 11 shows the behaviour of the energy contribution coefficient in each stage of the turbine as a function of the diameter ratio of the turbine runner. This graph shows that it is represented by a curve

$\left.\begin{array}{l}\xi_{1}=\frac{M_{e-1} \cdot \omega}{M_{e} \cdot \omega}=\frac{\left(R_{1} v_{1} \cos \alpha_{1}-R_{2} v_{2} \cos \alpha_{2}\right)}{\left(R_{1} v_{1} \cos \alpha_{1}-R_{1} v_{4} \cos \alpha_{4}\right)} \\ \xi_{2}=\frac{M_{e-1} \cdot \omega}{M_{e} \cdot \omega}=\frac{\left(R_{2} v_{3} \cos \alpha_{3}-R_{1} v_{4} \cos \alpha_{4}\right)}{\left(R_{1} v_{1} \cos \alpha_{1}-R_{1} v_{4} \cos \alpha_{4}\right)}\end{array}\right\}$

It is also true that:

$\xi_{1}+\xi_{2}=1$

Performing the analysis at the maximum hydraulic efficiency regime, we know that it is true that $v_{1}=2 u_{1} \cos \alpha_{1}, v_{4}=2 u_{4}$ $\cos \alpha_{4}, \alpha_{1}+\alpha_{2}=90^{\circ}$. Furthermore, from the velocity triangle we have to $v_{2}=u_{2} / \cos \alpha_{2}$. After taking these considerations into account in the above equation we obtain:

$\left.\begin{array}{l}\xi_{1}=\frac{1}{\cos 2 \alpha_{1}}\left(\cos ^{2} \alpha_{1}-\frac{1}{2}\left(\frac{D_{2}}{D_{1}}\right)^{2}\right) \\ \xi_{2}=\frac{1}{\cos 2 \alpha_{1}}\left(\frac{1}{2}\left(\frac{D_{2}}{D_{1}}\right)^{2}-\sin ^{2} \alpha_{1}\right)\end{array}\right\}$

decreasing (upper line) while $\xi_{2}$ by an increasing curve (lower line), in addition both must add a constant value equal to unity or $100 \%$. It can be seen that once a quotient value has been set, the values of $\xi_{1}$ and $\xi_{2}$ increase and decrease respectively as the value of the angle of attack $\alpha_{1}$ increases. Furthermore, it is observed that the values of the coefficients $\xi_{1}$ and $\xi_{2}$ will remain constant when increasing the angle of attack $\alpha_{1}$, only if the value of the diameter ratio $\frac{D_{2}}{D_{1}}$ increases correspondingly. 


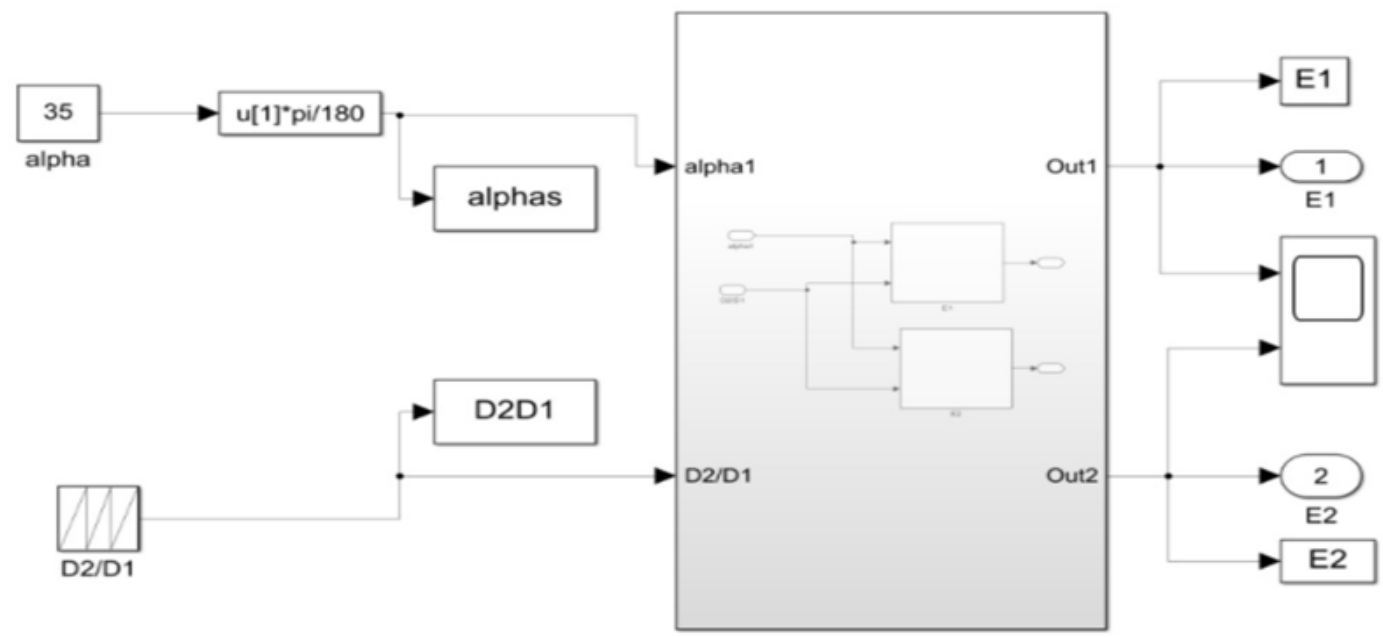

Fig. 10. MATLAB/Simulink for energy contribution expression

Considering the expressions, the diameter ratio and the expression of the maximum efficiency in the equations of $\xi_{1}$ and $\xi_{2}$, it is obtained that:

$\left.\begin{array}{l}\xi_{1}=\frac{\cos \alpha_{1}}{\cos 2 \alpha_{1}}\left(\cos \alpha_{1}-\sin \alpha_{1}\right) \\ \xi_{2}=\frac{\sin \alpha_{1}}{\cos 2 \alpha_{1}}\left(\cos \alpha_{1}-\sin \alpha_{1}\right)\end{array}\right\}$

With the considerations made, it has been possible to establish the coefficients of energy contribution per stage $\xi_{1}$ and $\xi_{2}$ as parameters solely dependent on the angle of attack $\alpha_{1}$. In this regard, it is possible to calculate the energy input in each stage of the cross-flow turbine only knowing the angle of entry of the flow. In the case of an angle $\alpha_{1}=15^{\circ}$, $78.88 \%$ are obtained as energy contributions in the first stage and $21.12 \%$ in the second stage, which is close to that obtained by Durgin and Fay who obtain $73 \%$ in the first stage and therefore $27 \%$ as energy contribution in the second stage [6].

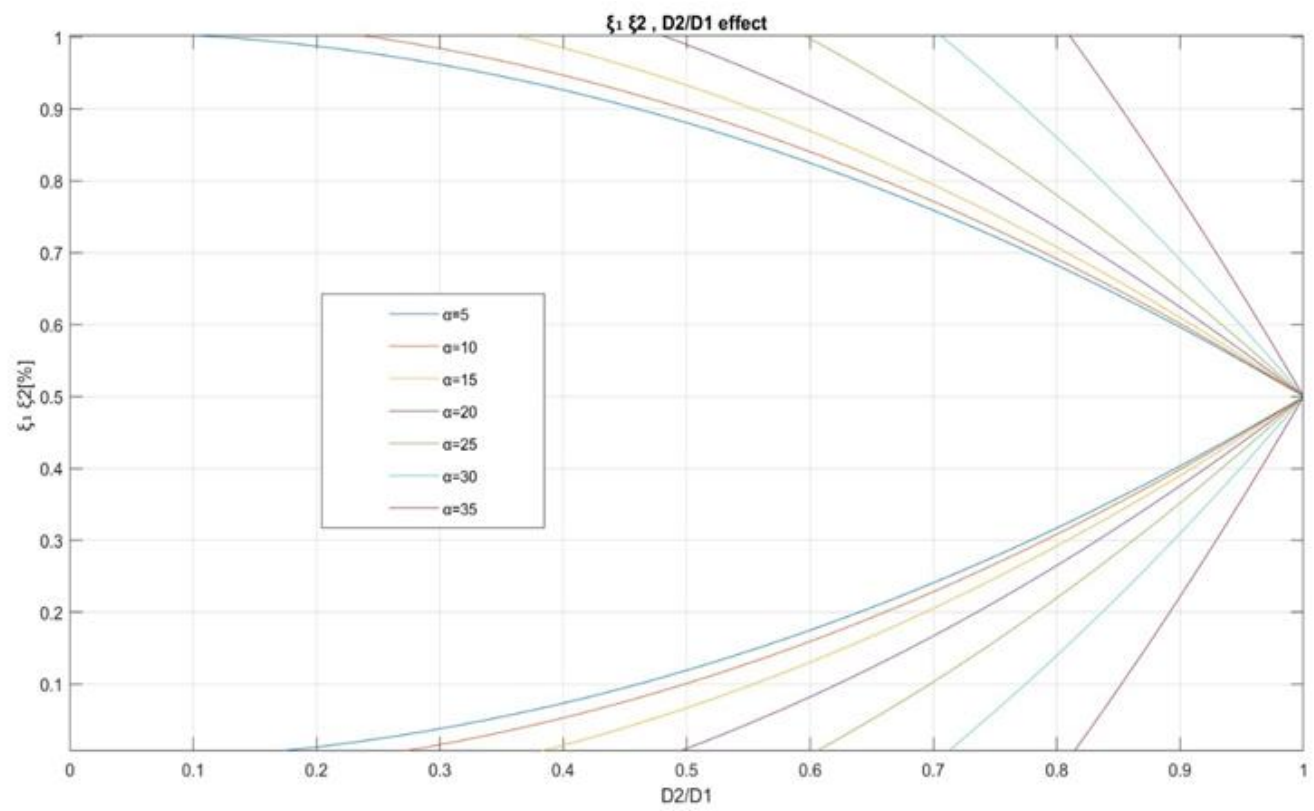

Fig. 11.: Energy contribution by stage as a function of $D_{2} / D_{1}$

\subsection{Effect of the $D_{2} / D_{1}$ Diameter Ratio}

Combining expressions (21), (27) and (28) in (13), we find that the maximum hydraulic efficiency is given by:

$$
\eta_{\mathrm{hmax}}=\frac{\varphi^{2}}{2}\left(1+\sqrt{1-\left(\frac{D_{2}}{D_{1}}\right)^{4}}\right)
$$

Figure 13 shows the behaviour of the maximum hydraulic efficiency achieved as a function of the variation of the runner diameter ratio after being solved by Simulink. It can also be seen in this graph that the maximum hydraulic efficiency is represented by a decreasing curve as the value of the diameter ratio increases. 


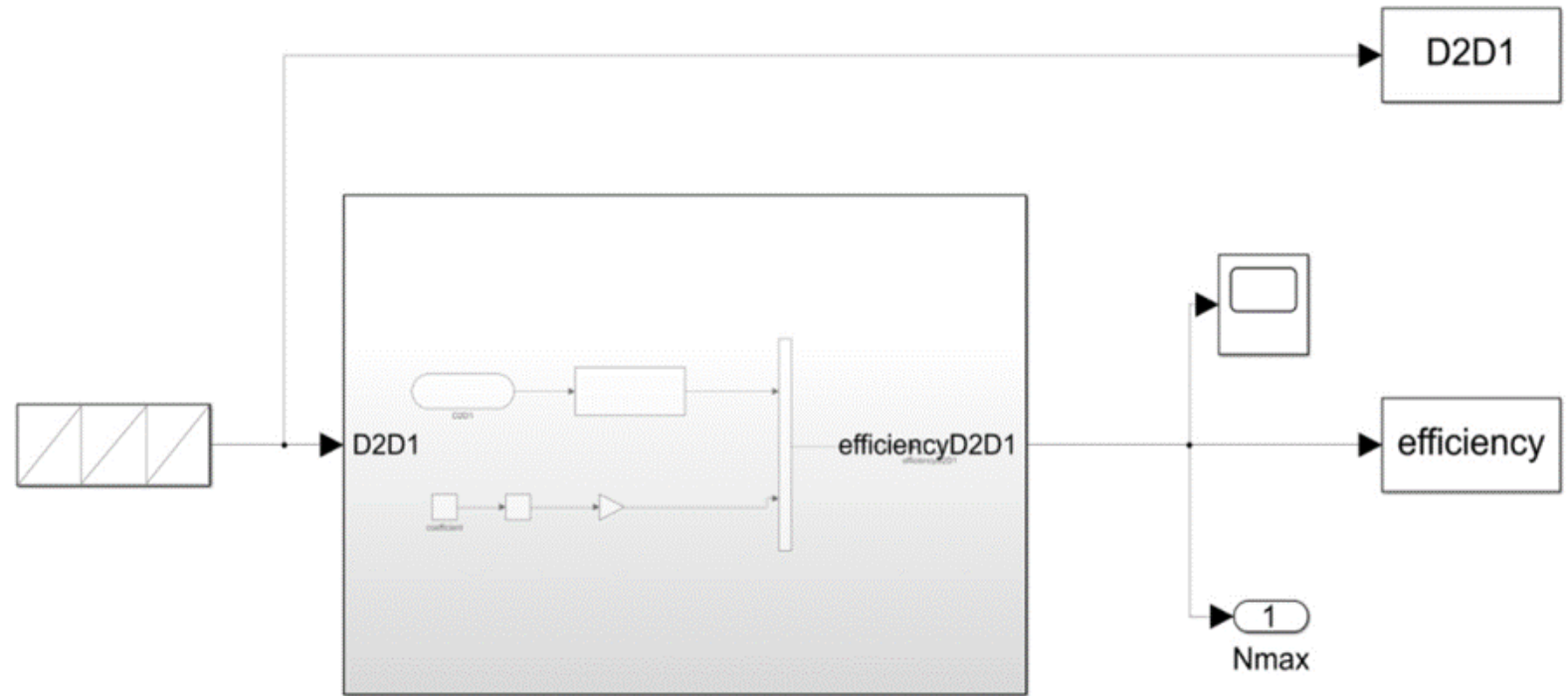

Fig. 12. MATLAB/Simulink for equation (28)

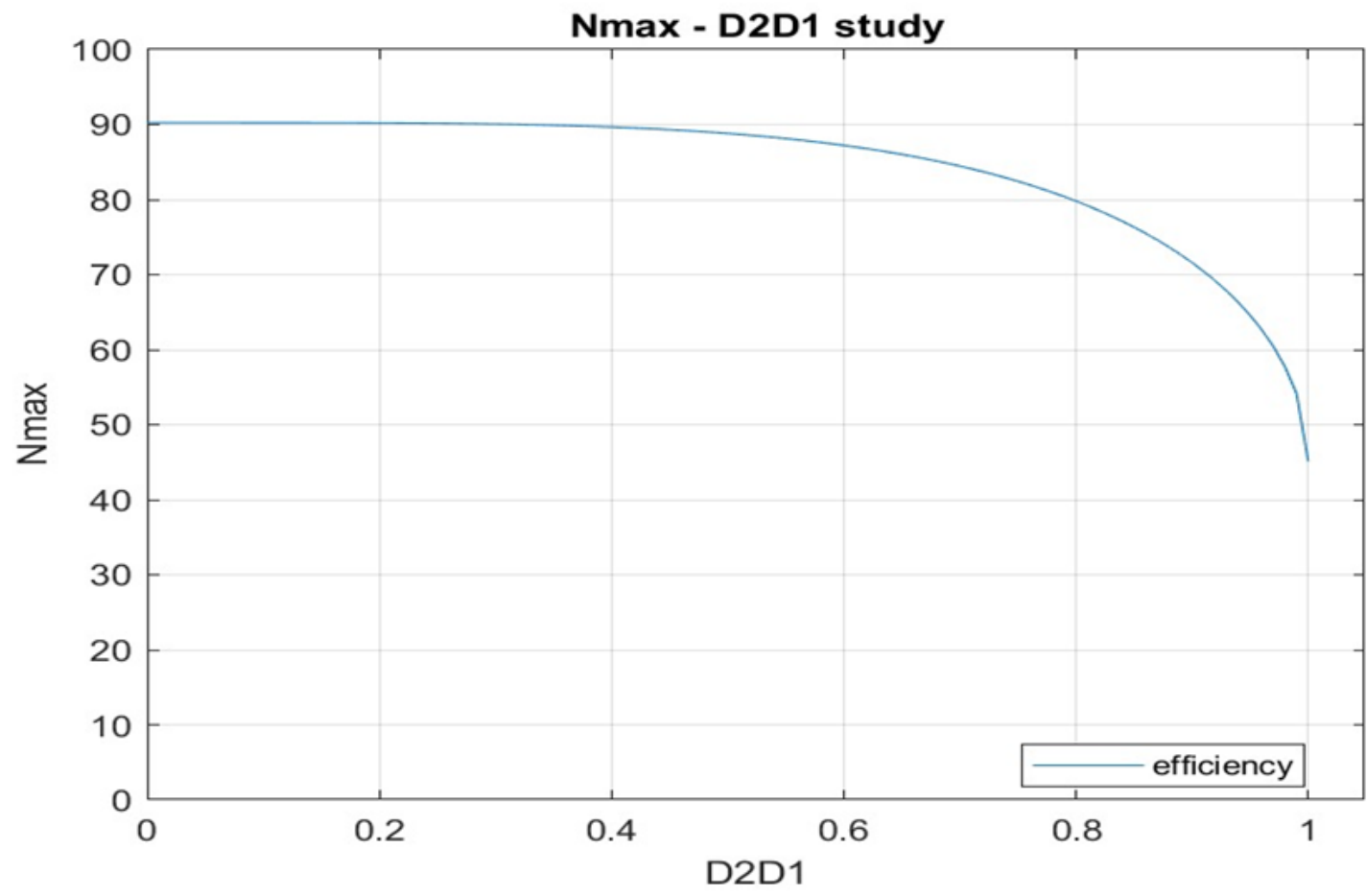

Fig. 13. Maximum Hydraulic Efficiency as a function of $D_{2} / D_{1}$

According to Desai and Aziz, when evaluating this type of turbines experimentally, they conclude that the higher the diameter ratio is, corresponds to a lower efficiency, and they also affirm that the maximum efficiency would be found in the vicinity of a diameter ratio of 0.68 [9]. When calculating the relation of diameters according to equation (27) for an angle $\alpha_{1}=15^{0}$, we have $\frac{D_{2}}{D_{1}}=0.707$, which is in accordance with what is maintained by these researchers. From Figures 11 and 13 it can be deduced that the higher the energy transferred in the first stage, the greater the efficiency of the turbine, which does not conform to reality, since a lower and lower value of diameter ratio implies an increase in $D_{1}-D_{2}$, which produces a wide difference between the inlet and outlet sections of each stage, causing considerable losses during the flow through the runner, seeking a decrease in efficiency [1]. In addition, large values of $\frac{D_{2}}{D_{1}}$ would make the turbine inefficient since the water jet would flow out of the space between blades in the inner periphery [9], therefore in Figure 8 we could consider with a good approximation that from $\frac{D_{2}}{D_{1}}$ $=0.66$ henceforth, the behaviour would be more in line with reality, as some researchers indicate that 0.66 is the optimal relationship between runner diameters [1]. 


\section{Conclusions and Recommendations}

A theoretical study based on the control volume method was developed and applied to the study of hydraulic efficiency in the runner of a cross flow turbine, concluding that:

The efficiency behaviour of the cross-flow turbine obtained by varying the values of some of its design parameters $\left(\alpha_{1}, \beta_{1}\right.$ and $\left.\frac{D_{2}}{D_{1}}\right)$ is confirmed by experimental studies by other authors. In the design of this type of turbine, it should be ensured that $\alpha_{1}$ does not exceed $20^{\circ}$, since the turbine would become inefficient.

The exit angle of the blade $\beta_{2}$ can be theoretically determined as $90^{\circ}$, which is also verified in experimental results [10]. In the design of the cross-flow turbine, it must be guaranteed that $\beta_{1}=2 \alpha_{1}$ and also that $\frac{D_{2}}{D_{1}}=\sqrt{\sin \beta_{1}}$, since the fulfillment of these conditions makes the hydraulic efficiency maximum. The impact of the many parameters that influence the efficiency of the cross-flow turbine (such as the number of blades, the thickness of the blades or the clearance between injector and runner, as well as the shape of the injector walls) is still under investigation. However, it is preponderant that for the measurement of its effects on the performance of the turbine, methods and / or theoretical criteria are devised that link these design parameters with the efficiency of the turbine, thus allowing the obtaining of their optimal values. Since the improvement of the performance of this hydraulic turbine will depend on the optimization of these parameters.

\section{Acknowledgment}

The author gratefully acknowledges the support and assistance given by the department of Energy Engineering systems technology especially Dr. OZCAN ATLAM.

\section{$\underline{\text { ORCID }}$}

N. H. J. Paul

0000-0003-3788-9467

\section{References}

[1] Renewables First. Crossflow Turbines [Online]. 2015. https://www.renewablesfirst.co.uk/hydropower/ hydropower-learning-centre/crossflow-turbines/. $\quad\left(25^{\text {th }}\right.$ of May 2021)

[2] De Andrade, J.; Curiel, C.; Kenyery, F.; Aguillón, O.; Vásquez, A.; Asuaje, M. Numerical investigation of the internal flow in a Banki turbine. Int. J. Rotating Mach. 2011, 2011, 841214.

[3] Fukutomi J. Senoo Y. and Nakase Y. 1991. Numerical Method of Flow through a Cross-Flow Runner. JSME International Journal: Series II, Vol. 34, No. 1. Japan. pp 44-51.

[4] Fukutomi J., Nakase Y., Ichimiya M. and Ebisu H. 1995. Unsteady Forces on a Blade in a Cross-Flow Turbine. JSME International Journal: Vol. 38, No. 3. Japan. pp 404-410.

[5] Yunus A. Çengel and John M. Cimbala; Fluid mechanics: Fundamentals and applications $1^{\text {st }}$ Ed 2006.

[6] Durgin, WW., and Fay WK. 1984. Some Fluid Characteristics of a Cross Flow Type Hydraulic Turbine. ASME Small Hydropower Fluid Machinery. USES.

[7] Choi, Y-D., Zhao, L. and Kurokawa, J., 2006. A study on the optimal configuration and performance Improvement of a Micro cross flow hydraulic Turbine. Journal of the Korean Society of Marine Engineering Vol 30. No 2. 2006.

[8] Mockmore, CA. and Merryfield, F. 1949. The Banki Water Turbine. Engineering Experiment Station. Oregon State College. USES.

[9] Desai, V. R. and Aziz, N. M., 1994. An Experimental Investigation of cross flow turbine Efficiency. Journal of Fluids Engineering. Vol 116. Pp 545-550 1944.

[10] Desai VR and Aziz NM, 1991. An Experimental Study of the Effect of Some Design Parameters on Crossflow Turbine Efficiency. Engineering Report. Department of Civil Engineering. Clemson University. USES.

Nomenclature

\begin{tabular}{|c|c|c|}
\hline D1, D2: outer and inner diameter of the runner $[\mathrm{m}]$. & $\eta_{\mathrm{h}}:$ hydraulic efficiency. & $\left|W_{r}\right|:$ friction work on the blades. \\
\hline B: runner width $[\mathrm{m}]$. & Z: number of blades. & $\mathrm{g}$ : acceleration of gravity $\left(9.81 \mathrm{~m} / \mathrm{s}^{2}\right)$. \\
\hline $\mathrm{H}$ : available head $[\mathrm{m}]$. & $u$ : tangential velocity $=\omega \mathrm{Ri}[\mathrm{m} / \mathrm{s}]$ & $\vec{n}:$ vector normal to control surfaces. \\
\hline$\varphi:$ Coefficient of losses in the injector. & $w$ : relative velocity $[\mathrm{m} / \mathrm{s}]$ & $\begin{array}{l}\vec{r} \text { : radial vector for the position of the fluid } \\
\text { particles. }\end{array}$ \\
\hline $\mathrm{n}$ : rotational speed of the runner [rpm]. & $v:$ absolute velocity $[\mathrm{m} / \mathrm{s}]$ & $\vec{V}:$ absolute velocity vector. \\
\hline $\mathrm{n}_{11}:$ unit rotational speed $=\mathrm{nD} 1 / \sqrt{H}$ & $\xi_{1}, \xi_{2}:$ energy contribution at each stage. & Subscripts: \\
\hline$\omega:$ angular velocity $[\mathrm{rad} / \mathrm{s}]$ & $\begin{array}{l}\alpha \text { : angle between absolute velocity and } \\
\text { tangential velocity. }\end{array}$ & 1: Entrance of the first stage. \\
\hline Q: water flow $\left[\mathrm{m}^{3} / \mathrm{s}\right]$. & $\begin{array}{l}\beta \text { : angle between relative velocity and } \\
\text { tangential velocity. }\end{array}$ & 2: Departure of the first stage. \\
\hline M: resultant moment in the axial direction $[\mathrm{Nm}]$. & $\begin{array}{l}\theta: \text { arc subtended by flux on the inner and } \\
\text { outer periphery of the runner. }\end{array}$ & 3: Entrance of the second stage. \\
\hline$\rho:$ density of the working fluid $\left[\mathrm{Kg} / \mathrm{m}^{3}\right]$. & $\mathrm{L}_{\mathrm{i}}$ : length of the arc that embraces the flow. & 4: Exit of the second stage. \\
\hline
\end{tabular}

